Article

\title{
Three New Highly Oxygenated Germacranolides from Carpesium Divaricatum and Their Cytotoxic Activity
}

\author{
Tao Zhang ${ }^{1}$, Jin-Guang $\mathrm{Si}^{1}{ }^{1}$, Qiu-Bo Zhang ${ }^{1}$, Jia-Huan Chen ${ }^{1,2}$, Gang Ding ${ }^{1}$, Hong-Wu Zhang ${ }^{1}$, \\ Hong-Mei Jia ${ }^{1}$ and Zhong-Mei Zou ${ }^{1, *}$ \\ 1 Institute of Medicinal Plant Development, Peking Union Medical College and Chinese Academy of Medical \\ Sciences, Beijing 100193, China; zt830423@163.com (T.Z.); sjgking@126.com (J.-G.S.); \\ zhangqiubolsh@163.com (Q.-B.Z.); chenjiahuan0107@163.com (J.-H.C.); gding@implad.ac.cn (G.D.); \\ 18101318775@163.com (H.-W.Z.); rainbow-grape@163.com (H.-M.J.) \\ 2 School of Traditional Chinese Medicine, Shenyang Pharmaceutical University, Shenyang 110016, China \\ * Correspondence: zmzou@implad.ac.cn; Tel.: +86-010-57833290
}

Received: 30 March 2018; Accepted: 27 April 2018; Published: 3 May 2018

check for updates

\begin{abstract}
Three new highly oxygenated (2-4), and two known (1 and 5) germacranolides, were isolated from the whole plant of Carpesium divaricatum. The planar structures and relative configurations of the new compounds were determined by detailed spectroscopic analysis. The absolute configuration of $\mathbf{1}$ was established using the circular dichroism (CD) method and X-ray diffraction, and the stereochemistry of the new compounds $\mathbf{2}-\mathbf{4}$ were determined using similar $\mathrm{CD}$ spectra with $\mathbf{1}$. The new compound $\mathbf{2}$ and the known compound 5 exhibited potent cytotoxicity against hepatocellular cancer (Hep G2) and human cervical cancer (HeLa) cells, superior to those of the positive control cis-platin.
\end{abstract}

Keywords: Carpesium divaricatum; germacranolides; absolute configuration; cytotoxicity

\section{Introduction}

The genus Carpesium (Asteraceae) includes 25 species worldwide, most of which are distributed across Asia and Europe, particularly in southwest China [1,2]. In China, Korea, and Japan, many Carpesium species have been used for the treatment of fevers, colds, bruises, and snake bites, due to their antipyretic, analgesic, vermifugic, hemostatic, detoxifying, and anti-inflammatory properties [2]. The genus is rich in diverse sesquiterpenoid lactones, such as eudesmanolides and germacranolides [2-6]. Previous investigations indicate that sesquiterpenoid lactones possessing an $\alpha$-methylene- $\gamma$-lactone moiety are cytotoxic to human cancer cells [2-7]. Recently, six sesquiterpenoid lactones with new skeletons displaying significantly cytotoxic activity were isolated from Carpesium plants [8,9].

Carpesium divaricatum Sieb.et Zucc is widely distributed in China, and is traditionally used for the treatment of fevers, colds, bruises, insect bites and inflammatory diseases [10-15]. Previous investigations of this plant reported the isolation of germacrane-type sesquiterpene lactones [4,14-17]. The parent nucleus of the germacranes contains a 10-membered ring with different post-modifications to produce diverse structural features. Many skeletal types of germacranolides with broad biological activities, such as cytotoxic and anti-inflammatory properties, have been isolated from the genera Carpesium, Inula and Allagopappus [5,6,11,15-26]. Our previous study led to the isolation, structural elucidation and analysis of the cytotoxic activity of eight germacranolides from this plant [27].

As a part of our ongoing search for new bioactive products from medicinal plants in China, three new, and two known highly oxygenated germacranolides representing other subtype (Figure 1), 
were isolated from the whole plant of $C$. divaricatum. In this paper, a structural elucidation and bioactive evaluation of these compounds is presented.

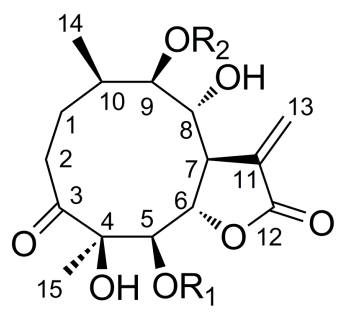<smiles>[R2]C(=O)CC(C)C</smiles>

Figure 1. Chemical structures of compounds 1-5.

\section{Results and Discussion}

\subsection{Purification of Compounds $\mathbf{1 - 5}$}

The whole plant of $C$. divaricatum was extracted three times with $\mathrm{EtOH}-\mathrm{H}_{2} \mathrm{O}$ (95:5). The five highly oxygenated germacranolides were isolated and purified via silica gel chromatograpy, Sephadex LH-20 gel chromatograpy, and semi-preparative High-Performance Liquid Chromatography (HPLC).

\subsection{Structure Elucidation of Compounds 1-5}

Compound 1 was identified as $4 \beta, 8 \alpha$-dihydroxy- $5 \beta$-isobutyryloxy- $9 \beta$-3-methylbutyryloxy-3-oxogermacran- $6 \alpha$, 12-olide (1), by comparison of its MS, NMR data, as well as optical rotation data, with reported data (supplementary materials Figure S1) [5]. However, its absolute configuration has not been determined. According to Beecham's rule, the CD spectrum (Figure 2) of $\mathbf{1}$ exhibited a positive Cotton effect near $254 \mathrm{~nm}$ ( $\alpha$-methylene- $\gamma$-lactone region), supporting $6 S, 7 R$ configuration [21]. Fortunately, a suitable crystal was obtained for X-ray diffraction to confirm the absolute configuration. The X-ray crystallographic analysis [flack parameter: 0.08(17)] established unambiguously the absolute configuration of 1 to be $4 R, 5 R, 6 S, 7 R, 8 R, 9 R$ and $10 R$ (Figure 3). Herein, the absolute configuration of 1 is reported for the first time. 

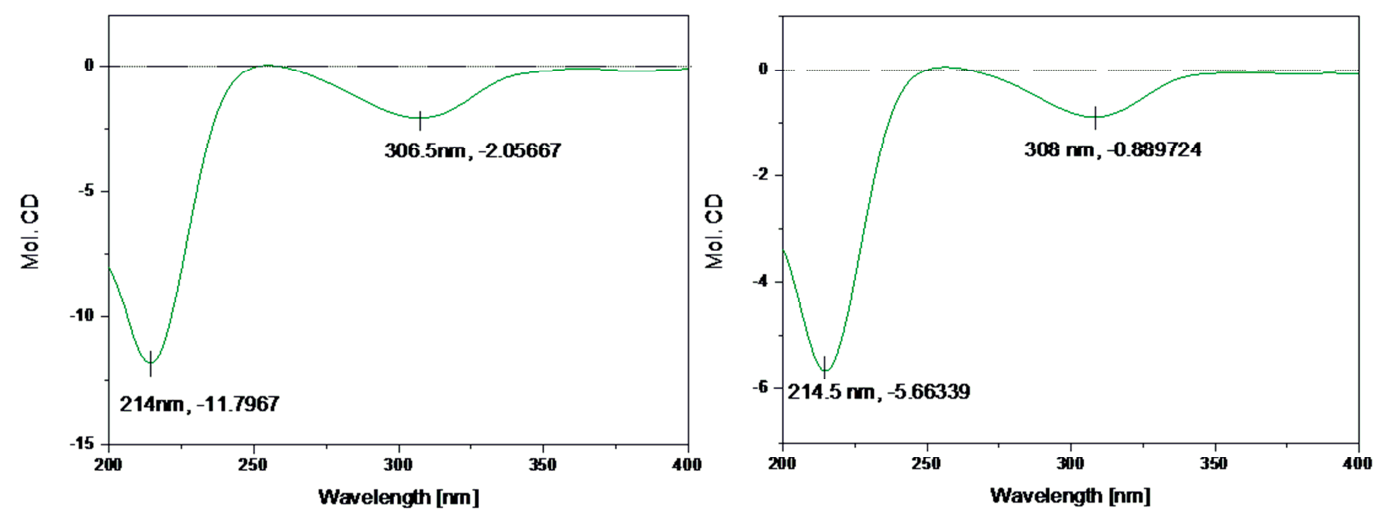

1

2

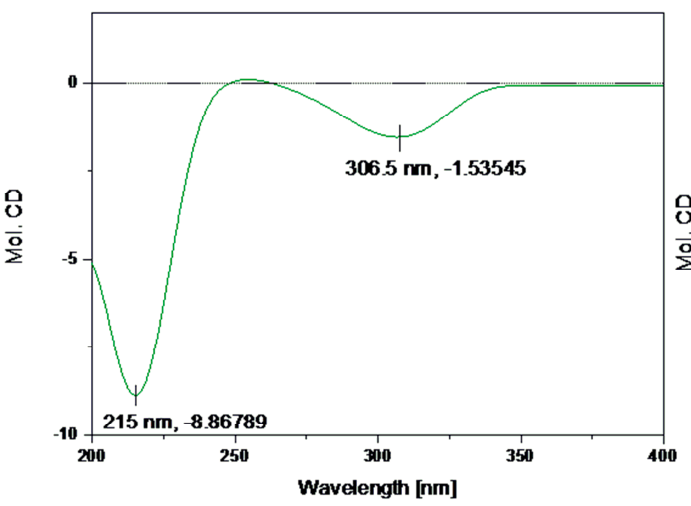

3

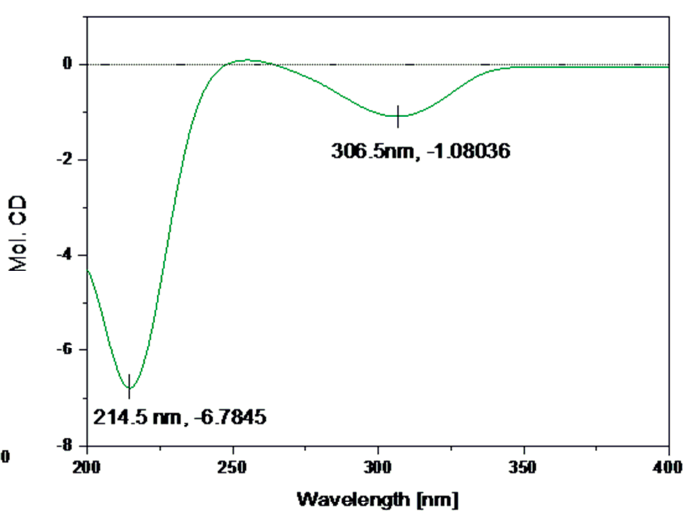

4

Figure 2. CD spectra of compounds 1-4.

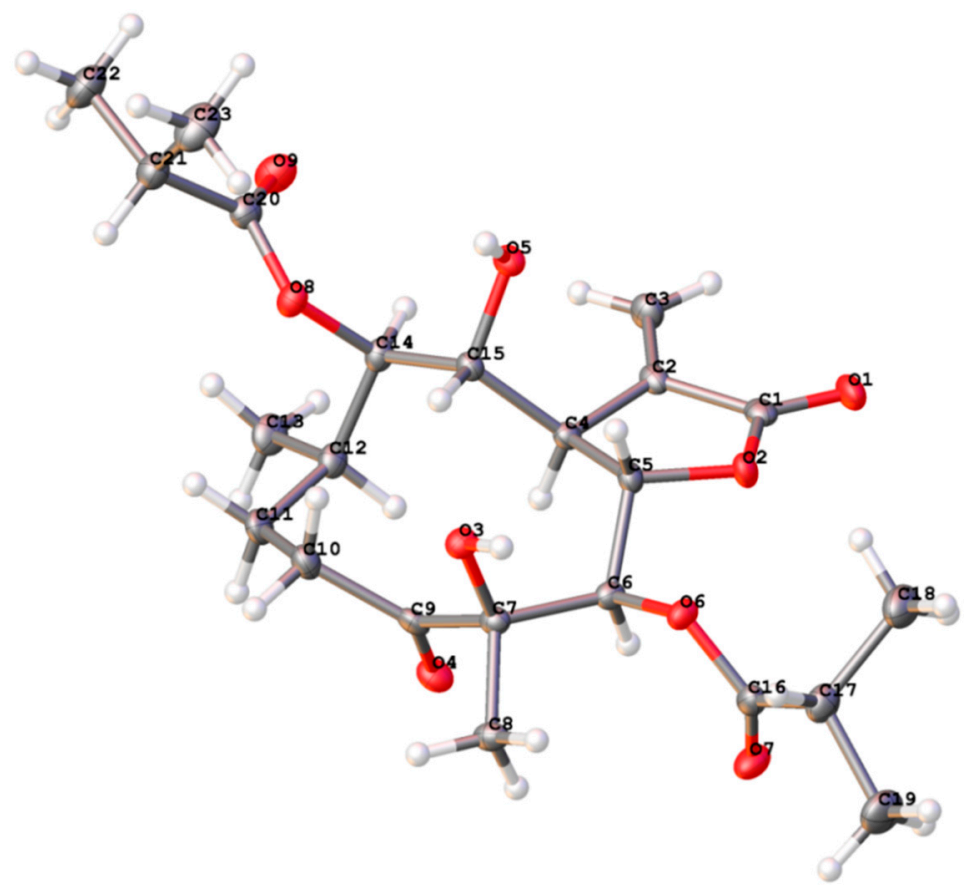

Figure 3. X-ray ORTEP drawing of $\mathbf{1 .}$

Compound 2 was obtained as white needles. The molecular formula was assigned as $\mathrm{C}_{25} \mathrm{H}_{38} \mathrm{O}_{9}$, on the basis of the positive-ion HRESIMS peak at $m / z 505.2427[\mathrm{M}+\mathrm{Na}]^{+}$, together with its ${ }^{1} \mathrm{H}$ 
and ${ }^{13} \mathrm{C}$ NMR data (Table 1). Its IR spectrum showed hydroxy $\left(3458 \mathrm{~cm}^{-1}\right)$ and carbonyl $(1744$ and $\left.1718 \mathrm{~cm}^{-1}\right)$ absorptions. The ${ }^{1} \mathrm{H}$ and ${ }^{13} \mathrm{C}$ NMR spectra of 2 showed an $\alpha$-methylene- $\gamma$-lactone at $\delta_{\mathrm{H}}$ $6.27(1 \mathrm{H}, \mathrm{d}, J=3.0 \mathrm{~Hz}, \mathrm{Ha}-13)$ and $5.62(1 \mathrm{H}, \mathrm{d}, J=3.0 \mathrm{~Hz}, \mathrm{Hb}-13), \delta_{\mathrm{C}} 132.6(\mathrm{C}-11), 123.9(\mathrm{C}-13)$ and 169.7 (C-12); three carbonyl carbons at $\delta_{\mathrm{C}} 217.8(\mathrm{C}-3), 172.5\left(\mathrm{C}-1^{\prime}\right)$ and $173.3\left(\mathrm{C}-1^{\prime \prime}\right)$; one oxygenated tertiary carbon at $80.3(\mathrm{C}-4)$; four oxygenated methines at $\delta_{\mathrm{H}} 5.36(1 \mathrm{H}, \mathrm{dd}, J=8.5,2.0 \mathrm{~Hz}, \mathrm{H}-5)$, $4.60(1 \mathrm{H}, \mathrm{dd}, J=8.5,5.0 \mathrm{~Hz}, \mathrm{H}-6), 4.35(1 \mathrm{H}, \mathrm{d}, J=10.5 \mathrm{~Hz}, \mathrm{H}-8)$, and $5.11(1 \mathrm{H}, \mathrm{d}, J=10.5 \mathrm{~Hz}, \mathrm{H}-9)$, $\delta_{\mathrm{C}} 78.2(\mathrm{C}-5), 79.8(\mathrm{C}-6), 70.3(\mathrm{C}-8)$, and 78.7 (C-9); and two methyl groups at $\delta_{\mathrm{H}} 0.92(3 \mathrm{H}, \mathrm{d}, J=7.0 \mathrm{~Hz}$, $\left.\mathrm{CH}_{3}-14\right), 1.18\left(3 \mathrm{H}, \mathrm{s}, \mathrm{CH}_{3}-15\right)$. These signals $\left({ }^{1} \mathrm{H}\right.$ and ${ }^{13} \mathrm{C}$ NMR data) implied that the structure of 2 was similar to that of $\mathbf{1}$, except that the isobutyryloxy group of $\mathbf{1}$ was replaced by a 3-methylbutyryloxy group at $\mathrm{C}-5$ in 2; this was further confirmed by the ${ }^{1} \mathrm{H}-{ }^{1} \mathrm{H}$ COSY, HSQC, and HMBC spectra (Figure 4). On the basis of these data, the planar structure of 2 was established.
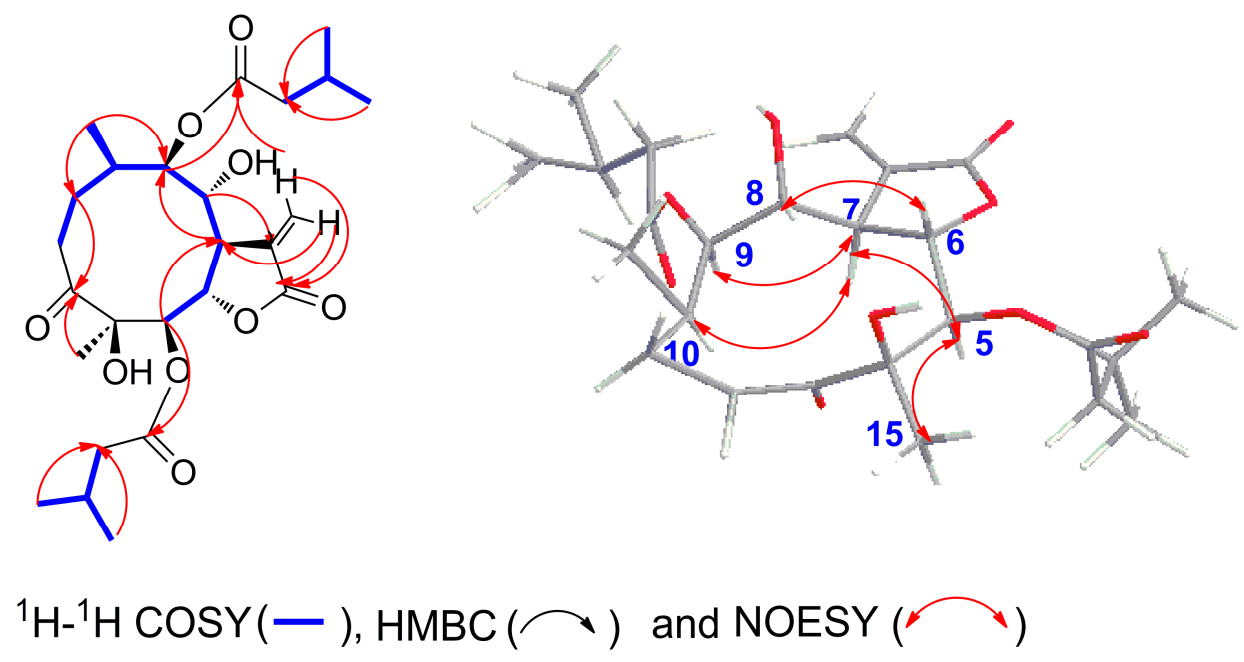

Figure 4. Key 2D correlations of compound 2.

The relative configuration of 2 was determined by analysis of ROESY data. The key NOE correlations of $\mathrm{H}-8 / \mathrm{H}-6, \mathrm{H}-7 / \mathrm{H}-10, \mathrm{H}-7 / \mathrm{H}-5, \mathrm{H}-7 / \mathrm{H}-9$, and $\mathrm{H}-5 / \mathrm{H}_{3}-15$ indicated that 2 had the same relative configuration as $\mathbf{1}$ (Figure 4). The CD spectrum of $\mathbf{2}$ showed positive Cotton effects at near $254 \mathrm{~nm}$, which closely resembled those of $\mathbf{1}$. Similar ROESY and CD data of $\mathbf{2}$ and $\mathbf{1}$ (Figure 2) assigned the absolute configuration of 2 as $4 R, 5 R, 6 S, 7 R, 8 R, 9 R$, and 10R. Thus, the structure of compound 2 , named divarolide $\mathrm{E}$, is defined as shown.

Compounds 3-4 possessed molecular formulas of $\mathrm{C}_{23} \mathrm{H}_{32} \mathrm{O}_{9}$ and $\mathrm{C}_{25} \mathrm{H}_{38} \mathrm{O}_{9}$, from their HRESIMS at $m / z 475.1939[\mathrm{M}+\mathrm{Na}]^{+}$and $m / z 505.2414[\mathrm{M}+\mathrm{Na}]^{+}$respectively. The ${ }^{1} \mathrm{H}$ and ${ }^{13} \mathrm{C}$ NMR data of 3-4 were similar to those of $\mathbf{1}$, except that the 2-methacryloyloxy group at C-9 in $\mathbf{3}$ was observed in place of 3-methylbutyryloxy group in 1, and an isobutyryloxy group at C-5 and the 3-methylbutyryloxy group at C-9 in 1 were replaced by two 2-methylbutyryloxy groups in 4, respectively. These observations were confirmed by analyses of relevant ${ }^{1} \mathrm{H}_{-}{ }^{1} \mathrm{H}$ COSY, HSQC and $\mathrm{HMBC}$ data (Table 1). The relative configurations of 3-4 were determined to be the same as those of $\mathbf{1}$, by comparison of ROESY data for relevant protons. Similar CD data of 3-4 and $\mathbf{1}$ (Figure 2) revealed the same absolute configurations of 3-4 as that of $\mathbf{1}$. Thus, the structures of compounds 3-4 were established as shown, and named divarolide $\mathrm{F}$ and divarolide $\mathrm{G}$ respectively.

The structure of the known compound (5) was identified as $4 \beta, 8 \alpha$-dihydroxy-5 $\beta$-2-methylbutyryloxy9 $\beta$-3-methylbutyryloxy-3-oxo-germacran-7 $\beta, 12 \alpha$-olide [5], by comparison of its spectroscopic data with reported data. 
Table 1. NMR spectral data of $\mathbf{2}-\mathbf{4}$.

\begin{tabular}{|c|c|c|c|c|c|c|}
\hline \multirow{2}{*}{ No. } & \multicolumn{2}{|r|}{$2^{a}$} & \multicolumn{2}{|r|}{$3^{b}$} & \multicolumn{2}{|r|}{$4^{a}$} \\
\hline & $\delta_{\mathrm{C}}$, Type & $\delta_{\mathrm{H}}(J$ in $\mathrm{Hz})$ & $\delta_{\mathrm{c}}$, Type & $\delta_{\mathrm{H}}(J$ in $\mathrm{Hz})$ & $\delta_{\mathrm{c}}$, Type & $\delta_{\mathrm{H}}(J$ in $\mathrm{Hz})$ \\
\hline 1 & $25.3 \mathrm{CH}_{2}$ & $1.79 \mathrm{~m}, 1.65 \mathrm{~m}$ & $25.3 \mathrm{CH}_{2}$ & $1.83 \mathrm{~m}, 1.71 \mathrm{~m}$ & $25.3 \mathrm{CH}_{2}$ & $1.87 \mathrm{~m}, 1.75 \mathrm{~m}$ \\
\hline 2 & $32.8 \mathrm{CH}_{2}$ & $3.76 \mathrm{br} \mathrm{d}(7.5), 2.22 \mathrm{~m}$ & $32.9 \mathrm{CH}_{2}$ & $3.81 \mathrm{br} \mathrm{d}(7.5), 2.16 \mathrm{~m}$ & $33.2 \mathrm{CH}_{2}$ & $3.87 \mathrm{~m}, 2.29 \mathrm{~m}$ \\
\hline 3 & $217.8 \mathrm{C}$ & & $217.6 \mathrm{C}$ & & $217.6 \mathrm{C}$ & \\
\hline 4 & $80.3 \mathrm{C}$ & & $80.4 \mathrm{C}$ & & $80.3 \mathrm{C}$ & \\
\hline 5 & $78.2 \mathrm{CH}$ & $5.36 \mathrm{dd}(8.5,2.0)$ & $78.1 \mathrm{CH}$ & $5.37 \mathrm{dd}(9.6,1.2)$ & $78.1 \mathrm{CH}$ & 5.39 br d (9.5) \\
\hline 6 & $79.8 \mathrm{CH}$ & $4.60 \mathrm{dd}(8.5,5.0)$ & $79.9 \mathrm{CH}$ & $4.65 \mathrm{dd}(9.6,6.0)$ & $79.9 \mathrm{CH}$ & $4.69 \mathrm{dd}(9.5,6.5)$ \\
\hline 7 & $41.5 \mathrm{CH}$ & $2.97 \mathrm{~m}$ & $41.6 \mathrm{CH}$ & $3.01 \mathrm{~m}$ & $41.7 \mathrm{CH}$ & $3.02 \mathrm{~m}$ \\
\hline 8 & $70.3 \mathrm{CH}$ & $4.35 \mathrm{~d}(10.5)$ & $70.3 \mathrm{CH}$ & $4.43 \mathrm{~d}(10.2)$ & $70.5 \mathrm{CH}$ & $4.40 \mathrm{~d}(10.0)$ \\
\hline 9 & $78.7 \mathrm{CH}$ & $5.11 \mathrm{~d}(10.5)$ & $79.3 \mathrm{CH}$ & $5.18 \mathrm{~d}(10.2)$ & $78.4 \mathrm{CH}$ & $5.15 \mathrm{~d}(10.0)$ \\
\hline 10 & $29.8 \mathrm{CH}$ & $2.15 \mathrm{~m}$ & $30.1 \mathrm{CH}$ & $2.21 \mathrm{~m}$ & $30.0 \mathrm{CH}$ & $2.23 \mathrm{~m}$ \\
\hline 11 & $132.6 \mathrm{C}$ & & $132.7 \mathrm{C}$ & & $132.7 \mathrm{C}$ & \\
\hline 12 & $169.6 \mathrm{C}$ & & $169.6 \mathrm{C}$ & & $169.5 \mathrm{C}$ & \\
\hline 13 & $123.9 \mathrm{CH}_{2}$ & $\begin{array}{l}6.27 \mathrm{~d}(3.0), \\
5.62 \mathrm{~d}(3.0)\end{array}$ & $123.8 \mathrm{CH}_{2}$ & $\begin{array}{l}6.30 \mathrm{~d}(3.0) \\
5.67 \mathrm{~d}(3.0) \\
\end{array}$ & $123.8 \mathrm{CH}_{2}$ & $\begin{array}{l}6.32 \mathrm{~d}(3.0) \\
5.67 \mathrm{~d}(3.0) \\
\end{array}$ \\
\hline 14 & $20.0 \mathrm{CH}_{3}$ & $0.92 \mathrm{~d}(7.0)$ & $19.9 \mathrm{CH}_{3}$ & $0.94 \mathrm{~d}(6.6)$ & $20.0 \mathrm{CH}_{3}$ & $0.98 \mathrm{~d}(6.5)$ \\
\hline 15 & $23.4 \mathrm{CH}_{3}$ & $1.18 \mathrm{~s}$ & $23.3 \mathrm{CH}_{3}$ & $1.21 \mathrm{~s}$ & $23.5 \mathrm{CH}_{3}$ & $1.24 \mathrm{~s}$ \\
\hline $1^{\prime}$ & $172.5 \mathrm{C}$ & & $176.3 \mathrm{C}$ & & $175.9 \mathrm{C}$ & \\
\hline $2^{\prime}$ & $42.7 \mathrm{CH}_{2}$ & $2.31 \mathrm{~d}(7.0), 2.26 \mathrm{o}$ & $33.9 \mathrm{CH}$ & $2.67 \mathrm{~m}$ & $41.3 \mathrm{CH}$ & $2.52 \mathrm{~m}$ \\
\hline $3^{\prime}$ & $25.3 \mathrm{CH}$ & $2.09 \mathrm{o}^{\mathrm{c}}$ & $18.0 \mathrm{CH}_{3}$ & $1.21 \mathrm{~d}(6.6)$ & $26.3 \mathrm{CH}_{2}$ & $1.76 \mathrm{o}, 1.52 \mathrm{o}$ \\
\hline $4^{\prime}$ & $21.3 \mathrm{CH}_{3}$ & $0.96 \mathrm{~d}(6.0)$ & $17.9 \mathrm{CH}_{3}$ & $1.20 \mathrm{~d}(6.6)$ & $16.1 \mathrm{CH}_{3}$ & $1.24 \mathrm{~d}(7.0)$ \\
\hline $5^{\prime}$ & $21.4 \mathrm{CH}_{3}$ & $0.95 \mathrm{~d}(6.0)$ & & & $10.7 \mathrm{CH}_{3}$ & $0.98 \mathrm{t}(7.0)$ \\
\hline $1^{\prime \prime}$ & $173.3 \mathrm{C}$ & & $167.2 \mathrm{C}$ & & $176.7 \mathrm{C}$ & \\
\hline $2^{\prime \prime}$ & $43.0 \mathrm{CH}_{2}$ & $2.26 \mathrm{o}, 2.06 \mathrm{o}$ & $136.4 \mathrm{C}$ & & $41.5 \mathrm{CH}$ & $2.52 \mathrm{~m}$ \\
\hline $3^{\prime \prime}$ & $25.4 \mathrm{CH}$ & $2.09 \mathrm{o}$ & $124.7 \mathrm{CH}_{2}$ & $\begin{array}{l}5.63 \mathrm{dq}(3.6,1.8), \\
6.13 \mathrm{dq}(3.6,1.8)\end{array}$ & $26.2 \mathrm{CH}_{2}$ & $1.76 \mathrm{o}, 1.52 \mathrm{o}$ \\
\hline $4^{\prime \prime}$ & $21.4 \mathrm{CH}_{3}$ & $0.96 \mathrm{~d}(6.5)$ & $17.1 \mathrm{CH}_{3}$ & $1.96 \mathrm{br} \mathrm{s}$ & $16.2 \mathrm{CH}_{3}$ & $1.26 \mathrm{~d}(7.0)$ \\
\hline $5^{\prime \prime}$ & $21.4 \mathrm{CH}_{3}$ & $0.95 \mathrm{~d}(6.5)$ & & & $10.6 \mathrm{CH}_{3}$ & $0.96 \mathrm{t}(7.0)$ \\
\hline
\end{tabular}

a Measured at $500 \mathrm{MHz}$ in methanol- $d_{4} ;{ }^{\mathrm{b}}$ Measured at $600 \mathrm{MHz}$ in methanol- $d_{4} ;{ }^{\mathrm{c}}$ Overlapped with other signals.

\subsection{In Vitro Cytotoxic Activities of Compounds $\mathbf{1 - 5}$}

Compounds 1-5 were evaluated for their cytotoxic activity against human cervical cancer (HeLa), hepatocellular cancer (Hep G2), and lung cancer (A549) cell lines (Table 2). The new compound 2, and the known compound 5, exhibited cytotoxicity against Hep G2 ( $\mathrm{IC}_{50}$ values of $\left.7.47 \mu \mathrm{M}\right)$ and HeLa $\left(\mathrm{IC}_{50}\right.$ values of $16.82 \mu \mathrm{M}$ ) cell lines, and the $\mathrm{IC}_{50}$ values were lower than those of the positive control cis-platin ( $\mathrm{IC}_{50}$ values of 13.03, and $15.34 \mu \mathrm{M}$ respectively). In addition, $\mathbf{1}$ and $\mathbf{2}$ also displayed strong cytotoxicity against Hep G2, with an $\mathrm{IC}_{50}$ value of $16.98 \mu \mathrm{M}$, and HeLa with an $\mathrm{IC}_{50}$ value of $16.82 \mu \mathrm{M}$.

Table 2. In Vitro Cytotoxic Activities of Compounds 1-5.

\begin{tabular}{cccc}
\hline \multirow{2}{*}{ Compounds } & \multicolumn{3}{c}{ IC $_{\mathbf{5 0}}(\boldsymbol{\mu M})$} \\
\cline { 2 - 4 } & $\mathbf{A 5 4 9}$ & HepG2 & Hela \\
\hline $\mathbf{1}$ & $>40$ & $16.98 \pm 2.23$ & $29.39 \pm 0.17$ \\
$\mathbf{2}$ & $30.70 \pm 0.51$ & $7.47 \pm 0.21$ & $16.82 \pm 0.27$ \\
$\mathbf{3}$ & $>40$ & $>40$ & $>40$ \\
$\mathbf{4}$ & $>40$ & $>40$ & $>40$ \\
$\mathbf{5}$ & $>40$ & $31.64 \pm 0.16$ & $11.63 \pm 1.00$ \\
cis-platin & $7.90 \pm 0.23$ & $13.03 \pm 1.49$ & $15.34 \pm 0.35$ \\
\hline
\end{tabular}

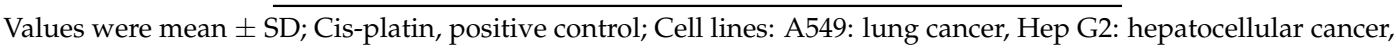
and HeLa: cervical cancer. 


\section{Materials and Methods}

\subsection{General Experimental Procedures}

Optical rotations were measured on a Perkin-Elmer 241 polarimeter (Perkin-Elmer, Waltham, MA, USA), and UV spectra were recorded on Shimadzu UV-2501 PC (Shimadzu, Kyoto, Japan). IR data were recorded using a Shimadzu FTIR-8400S spectrophotometer (Shimadzu, Kyoto, Japan). ${ }^{1} \mathrm{H}$ and ${ }^{13} \mathrm{C}$ NMR data were acquired using Bruker 500 and Bruker 600 instruments (Bruker, Rheinstetten, Germany), with solvent signals ( $\mathrm{CD}_{3} \mathrm{OD}: \delta_{\mathrm{H}} 3.30 / \delta_{\mathrm{C}} 49.0 \mathrm{ppm}$;) as references. HRESIMS data were acquired using a Q-TOF analyzer in SYNAPT HDMS system (Waters, Milford, MA, USA). X-ray diffraction data were collected on the Agilent GEMINI ${ }^{\mathrm{TM}} \mathrm{E}$ instrument (CrysAlisPro software, Version 1.171.35.11; Agilent, Santa Clara, CA, USA). HPLC was performed using Waters 2535 system (Waters, Milford, MA, USA), with the following components: preparative column, a Daisogel-C $18-100 \mathrm{~A}(10 \mu \mathrm{m}, 30 \times 250 \mathrm{~mm}$, ChuangXinTongHeng Sci. \& Tech., Beijing, China) and a YMC-Pack ODS-A column (5 $\mu \mathrm{m}, 10 \times 250 \mathrm{~mm}$, YMC, Kyoto, Japan); and detector, Waters 2489 UV. Sephadex LH-20 (40-70 $\mu$, Pharmacia Biotech AB, Uppsala, Sweden), silica gel (60-100, 100-200 and 200-300 mesh) and silica gel GF254 sheets (0.20-0.25 mm) (Qingdao Marine Chemical Plant, Qingdao, China) were used for column chromatography and TLC, respectively. TLC spots were visualized under UV light and by dipping into $5 \% \mathrm{H}_{2} \mathrm{SO}_{4}$ in EtOH, followed by heating.

\subsection{Plant Material}

The whole plants of $C$. divaricatum were collected from EnShi, Hubei province (China) in August of 2013. They were identified by Prof. Ben-Gang Zhang of Institute of Medicinal Plant Development. A voucher specimen (No. 20130828) was deposited in the National Compound Library of Traditional Chinese Medicines, Institute of Medicinal Plant Development, Chinese Academy of Medical Sciences \& Peking Union Medical College (CAMS \& PUMC), Beijing, China.

\subsection{Isolation and Purification of Compounds 1-5}

The air-dried plants $\left(9 \mathrm{~kg}\right.$ ) were extracted three times (7 days each time) with $\mathrm{EtOH}-\mathrm{H}_{2} \mathrm{O}(95: 5)$ at room temperature. The combined extract was concentrated under reduced pressure to furnish a dark brown residue $\left(570 \mathrm{~g}\right.$ ), which was suspended in $\mathrm{H}_{2} \mathrm{O}$ and partitioned in turn with petroleum ether (bp 60-90 ${ }^{\circ} \mathrm{C}$ ), EtOAc, and n-BuOH. The EtOAc extract (207 g) was separated chromatographically on silica gel column $(60-100$ mesh, $16 \times 20 \mathrm{~cm})$ with a gradient mixture of $\mathrm{CH}_{2} \mathrm{Cl}_{2}-\mathrm{MeOH}(100: 1,60: 1$, 30:1, 15:1 and 6:1) as eluent. Five fractions were collected according to TLC analysis. Fraction A $\left(\mathrm{CH}_{2} \mathrm{Cl}_{2}-\mathrm{MeOH}, 100: 1,140 \mathrm{~g}\right)$ was separated by silica gel column chromatography (CC) (100-200 mesh, $16 \times 20 \mathrm{~cm})$ with petroleum ether-aceton $(50: 1,25: 1,20: 1,15: 1,12: 1,10: 1,7: 1,5: 1,3: 1$ and 1:1) as eluent to give fractions $A_{1}-A_{11}$. Fraction $A_{10}$ (petroleum ether-aceton, 3:1, $40 \mathrm{~g}$ ) was separated by Sephadex LH-20 CC $(5 \times 200 \mathrm{~cm}, \mathrm{MeOH})$ to give Fr. $\mathrm{A}_{10} \mathrm{~S}_{1}-\mathrm{Fr} . \mathrm{A}_{10} \mathrm{~S}_{3}$. Fraction $\mathrm{A}_{10} \mathrm{~S}_{2}(20 \mathrm{~g})$ was then subjected to MCI gel CC $(6 \times 50 \mathrm{~cm})$ with a gradient mixture of $\mathrm{MeOH}-\mathrm{H}_{2} \mathrm{O}(60: 40,80: 20$, and 100:0, $4000 \mathrm{~mL}$ each) to give three fractions (Fr. $\mathrm{A}_{10} \mathrm{~S}_{2} \mathrm{M}_{1}-\mathrm{Fr} . \mathrm{A}_{10} \mathrm{~S}_{2} \mathrm{M}_{3}$ ).

Fraction $\mathrm{A}_{10} \mathrm{~S}_{2} \mathrm{M}_{2}$ (13 g) was further separated chromatographically on silica gel column (200-300 mesh, $5 \times 50 \mathrm{~cm}$ ) with a gradient mixture of $\mathrm{CH}_{2} \mathrm{Cl}_{2}-\mathrm{MeOH}(150: 1,100: 1,50: 1$ and 20:1) as eluent, and a total of 86 fractions (Fr. $\mathrm{A}_{10} \mathrm{~S}_{2} \mathrm{M}_{2}-1-86,200 \mathrm{~mL}$ each) were collected. Fraction $\mathrm{A}_{10} \mathrm{~S}_{2} \mathrm{M}_{2}-34-50$ (1.5 g) were separated by preparative $\mathrm{HPLC}\left(20 \mathrm{~mL} / \mathrm{min}, 70 \% \mathrm{MeOH}\right.$ in $\left.\mathrm{H}_{2} \mathrm{O}\right)$ and semipreparative HPLC (2 mL/min, 52-75\% MeOH in $\mathrm{H}_{2} \mathrm{O}$ for $25 \mathrm{~min}$ and followed by 75-95\% $\mathrm{MeOH}$ in $\mathrm{H}_{2} \mathrm{O}$ for 10 min; $2 \mathrm{~mL} / \mathrm{min}, 40-80 \% \mathrm{MeCN}$ in $\mathrm{H}_{2} \mathrm{O}$ for $40 \mathrm{~min}$ ) to yield 3 (5 mg). Fraction $\mathrm{A}_{10} \mathrm{~S}_{2} \mathrm{M}_{2}-74-79$ (140 mg) were purified using semipreparative HPLC $\left(2 \mathrm{~mL} / \mathrm{min}, 60-80 \% \mathrm{MeOH}\right.$ in $\mathrm{H}_{2} \mathrm{O}$ for $25 \mathrm{~min}$ and followed by 80-90\% MeOH in $\mathrm{H}_{2} \mathrm{O}$ for $20 \mathrm{~min} ; 2 \mathrm{~mL} / \mathrm{min}, 30-70 \% \mathrm{MeCN}$ in $\mathrm{H}_{2} \mathrm{O}$ for $40 \mathrm{~min}$ ) and to yield 1 (30 mg).

Fraction $\mathrm{A}_{9}$ (petroleum ether-Aceton, 5:1, $30 \mathrm{~g}$ ) was separated by Sephadex LH-20 CC $(5 \times 200 \mathrm{~cm}, \mathrm{MeOH})$ to give Fr. $\mathrm{A}_{9} \mathrm{~S}_{1}-\mathrm{Fr} . \mathrm{A}_{9} \mathrm{~S}_{3}$. Fraction $\mathrm{A}_{9} \mathrm{~S}_{2}(20 \mathrm{~g})$ was then subjected to MCI gel CC $(6 \times 50 \mathrm{~cm})$ with a gradient mixture of $\mathrm{MeOH}-\mathrm{H}_{2} \mathrm{O}(60: 40,80: 20$, and 100:0, $4000 \mathrm{~mL}$ each $)$ to give three 
fractions (Fr. $\mathrm{A}_{9} \mathrm{~S}_{2} \mathrm{M}_{1}-\mathrm{Fr} . \mathrm{A}_{9} \mathrm{~S}_{2} \mathrm{M}_{3}$ ). Fraction $\mathrm{A}_{9} \mathrm{~S}_{2} \mathrm{M}_{2}(10 \mathrm{~g})$ was further separated chromatographically on a silica gel column $(100-200$ mesh, $5 \times 50 \mathrm{~cm})$, with a gradient mixture of petroleum ether-Aceton (10:1, 7:1, 5:1, 3.5:1, 2:1 and 1:1) as eluent; a total of 200 fractions (Fr. $\mathrm{A}_{9} \mathrm{~S}_{2} \mathrm{M}_{2}-1-200,50 \mathrm{~mL}$ each) were collected. Fraction $\mathrm{A}_{9} \mathrm{~S}_{2} \mathrm{M}_{2}-107-112$ (2.5 g) were separated by silica gel column chromatography (CC) (200-300 mesh, $5 \times 40 \mathrm{~cm}$ ) with $\mathrm{CH}_{2} \mathrm{Cl}_{2}-\mathrm{MeOH}$ (150:1, 75:1, 30:1, and 15:1) as eluent to give Fr. $\mathrm{A}_{9} \mathrm{~S}_{2} \mathrm{M}_{2}-107-112-\mathrm{A}_{1}-\mathrm{Fr}$. $\mathrm{A}_{9} \mathrm{~S}_{2} \mathrm{M}_{2}-107-112-\mathrm{A}_{8}$. Fraction $\mathrm{A}_{9} \mathrm{~S}_{2} \mathrm{M}_{2}-107-112-\mathrm{A}_{3}\left(\mathrm{CH}_{2} \mathrm{Cl}_{2}-\mathrm{MeOH}, 75: 1\right.$, $500 \mathrm{mg}$ ) was further purified using semipreparative HPLC $\left(2 \mathrm{~mL} / \mathrm{min}, 65-90 \% \mathrm{MeOH}\right.$ in $\mathrm{H}_{2} \mathrm{O}$ for $40 \mathrm{~min} ; 2 \mathrm{~mL} / \mathrm{min}, 40-80 \% \mathrm{MeCN}$ in $\mathrm{H}_{2} \mathrm{O}$ for $40 \mathrm{~min}$ ) to yield 2 (4.5 mg), 4 (5 mg) and 5 (10 mg).

\subsection{Characterization of Compounds $\mathbf{2}-\mathbf{4}$}

Divarolide E (2): white needles $\left(\mathrm{CH}_{3} \mathrm{OH}\right),[\alpha]_{\mathrm{D}}^{20}-95.2$ (c $\left.0.125, \mathrm{MeOH}\right) ; \mathrm{UV}(\mathrm{MeOH}) \lambda \max (\log \varepsilon)$ : 210 (3.38) nm, IR (KBr) $v_{\max }: 3458,1744,1718,1661 \mathrm{~cm}^{-1}$; CD (MeOH) $215(\Delta \varepsilon-0.083), 308(\Delta \varepsilon-0.013)$ nm; HRESIMS (pos.): $m / z 505.2427[\mathrm{M}+\mathrm{Na}]^{+}$(calcd for $\left.\mathrm{C}_{25} \mathrm{H}_{38} \mathrm{O}_{9} \mathrm{Na}, 505.2414\right) ;{ }^{1} \mathrm{H}$ NMR and ${ }^{13} \mathrm{C}$ NMR data, see Table 1.

Divarolide F (3): white needles $\left(\mathrm{CH}_{3} \mathrm{OH}\right),[\alpha]_{\mathrm{D}}^{20}-78.7$ (c 0.150, MeOH); UV (MeOH) $\lambda \max (\log \varepsilon)$ : $200(4.68) \mathrm{nm}, \mathrm{IR}$ (neat) $v_{\max }: 3463,1762,1707,1647 \mathrm{~cm}^{-1}$; CD (MeOH) $215(\Delta \varepsilon-0.122), 307(\Delta \varepsilon-0.021)$ nm; HRESIMS (pos.): $m / z 475.1939[\mathrm{M}+\mathrm{Na}]^{+}$(calcd for $\mathrm{C}_{23} \mathrm{H}_{32} \mathrm{O}_{9} \mathrm{Na}, 475.1944$ ); ${ }^{1} \mathrm{H}$ NMR and ${ }^{13} \mathrm{C}$ NMR data, see Table 1.

Divarolide G (4): white needles $\left(\mathrm{CH}_{3} \mathrm{OH}\right),[\alpha]_{\mathrm{D}}^{20}-84.7$ (c 0.085, $\left.\mathrm{MeOH}\right) ; \mathrm{UV}(\mathrm{MeOH}) \lambda \max (\log \varepsilon)$ : $209(4.00) \mathrm{nm}, \mathrm{IR}$ (neat) $v_{\max }: 3440,2969,1740,1660 \mathrm{~cm}^{-1}$; CD (MeOH) $215(\Delta \varepsilon-0.099), 307(\Delta \varepsilon-0.016)$ nm; HRESIMS (pos.): $m / z 505.2414[\mathrm{M}+\mathrm{Na}]^{+}$(calcd for $\mathrm{C}_{25} \mathrm{H}_{38} \mathrm{O}_{9} \mathrm{Na}, 505.2414$ ); ${ }^{1} \mathrm{H}$ NMR and ${ }^{13} \mathrm{C}$ NMR data, see Table 1.

\subsection{X-ray Crystal Structure Analysis of Compound 1}

X-ray diffraction data were collected on the Agilent GEMINI ${ }^{\mathrm{TM}} \mathrm{E}$ instrument (CrysAlisPro software, Version 1.171.35.11), with enhanced $\mathrm{Cu} \mathrm{K} \alpha$ radiation $(\lambda=1.54184 \AA$ ). The structure was solved by direct methods and refined by full-matrix least-squares techniques (SHELXL-97). All non-hydrogen atoms were refined with anisotropic thermal parameters. Hydrogen atoms were located by geometrical calculations and from positions in the electron density maps. Crystallographic data (excluding structure factors) for $\mathbf{1}$ in this paper has been deposited with the Cambridge Crystallographic Data Centre (deposition number CCDC 1570798). Copies of the data can be obtained, free of charge, on application to CCDC, 12 Union Road, Cambridge CB2 1EZ, UK (fax: +44-12-23336033 or e-mail: deposit@ccdc.cam.ac.uk).

A colorless monoclinic crystal $(0.22 \times 0.18 \times 0.03 \mathrm{~mm})$ of 1 was grown from $\mathrm{MeOH}-\mathrm{H}_{2} \mathrm{O}$ (3:1). Crystal data: $\mathrm{C}_{24} \mathrm{H}_{36} \mathrm{O}_{9}, M=471.55, T=106.8 \mathrm{~K}$, triclinic, space groupP21, a $=14.2950(7)$ $\AA, \mathrm{b}=9.5219(4) \AA, \mathrm{c}=18.7748(11) \AA, \alpha=90.00^{\circ}, \beta=104.713^{\circ}, \gamma=90.00^{\circ}, V=2471.7(2) \AA^{3}, \mathrm{Z}=4$, $\rho=1.267 \mathrm{mg} / \mathrm{mm}^{3}, \mu(\mathrm{Cu} \mathrm{K} \alpha)=0.805 \mathrm{~mm}^{-1}$, measured reflections $=18092$, unique reflections $=9353$ $\left(R_{\text {int }}=0.0470\right)$, largest difference peak $/$ hole $=0.363 /-0.247$ e $\AA^{-3}$, and flack parameter $=0.08(17)$. The final Rindexes $[I>2 \sigma(I)]$ were $\mathrm{R}_{1}=0.0535$, and $w \mathrm{R}_{2}=0.1288$. The final Rindexes (all data) were $\mathrm{R}_{1}=0.0658$, and $\mathrm{wR}_{2}=0.1390$. The goodness of fit on $\mathrm{F}^{2}$ was 1.007 .

\subsection{Cytotoxicity Assays of Compounds 1-5}

Cell cultures: Human A549, HepG2, and HeLa cell lines from Cancer Institute and Hospital of Chinese Academy of Medical Sciences (Beijing, China), were cultured in Dulbecco's modified Eagle's medium (DMEM, Gibco, CA, USA) supplemented with 10\% $(v / v)$ fetal calf serum (Gibco, CA, USA), penicillin $\mathrm{G}$ (Macgene, China) 100 units $\mathrm{mL}^{-1}$ and streptomycin (Macgene, China) $100 \mu \mathrm{g} \mathrm{mL} \mathrm{L}^{-1}$, at $37^{\circ} \mathrm{C}$ under $5 \% \mathrm{CO}_{2}$.

Cell viability assay: The assay was run in triplicate. In a 96-well plate, each well was plated with $2 \times 10^{4}$ cells. After cell attachment overnight, the medium was removed, and each well was treated with $100 \mu \mathrm{L}$ of medium containing $0.1 \%$ DMSO or different concentrations of 
the test compounds and the positive control cis-platin. The plate was incubated at $37{ }^{\circ} \mathrm{C}$ for 4 days in a humidified, $5 \% \mathrm{CO}_{2}$ atmosphere. Cytotoxicity was determined using a modified 3-(4,5-dimethylthiazol-2-yl)-2,5-diphenyltetrazolium bromide (MTT) colorimetric assay [28]. After the addition of a $10 \mu \mathrm{L}$ MTT solution $(5 \mathrm{mg} / \mathrm{mL})$, cells were incubated at $37^{\circ} \mathrm{C}$ for $4 \mathrm{~h}$. After adding $150 \mu \mathrm{L}$ DMSO, cells were shaken to mix thoroughly. The absorbance of each well was measured at $540 \mathrm{~nm}$ in a Multiscan photometer. The $\mathrm{IC}_{50}$ values were calculated by Origin software and listed in Table 2.

Statistical analysis: Values were expressed as mean \pm SD. Statistical analyses were performed using the Student's $t$-test. Differences were considered significant when associated with a probability of $5 \%$ or less $(p \leq 0.05)$.

\section{Conclusions}

In conclusion, three new compounds (2-4), as well as two known compounds (1 and 5), were isolated from the whole plant of C. divaricatum. Structurally, all compounds contained a 5 -membered $\gamma$-lactone ring fused to a circular 10-membered carbocycle. We obtained a set of isomers $(2 / 4 / 5)$ from the same plant. The isolation of these isomers is a huge challenge because they are highly oxygenated and have similar structures. The absolute configuration of compound $\mathbf{1}$ was unambiguously established by X-ray diffraction. The other compounds with the same skeleton were determined by comparison of NOESY and CD data with those of $\mathbf{1}$. Compounds 1 and 5 showed significant cytotoxicity against two human tumor cell lines. These findings are an important addition to the present knowledge on the structurally diverse and biologically significant germacranolide family.

Supplementary Materials: The Supplementary Materials are available online.

Author Contributions: T.Z. and Z.-M.Z. conceived and designed the experiments; T.Z., Q.-B.Z., J.-G.S., J.-H.C., and G.D. performed the experiments; H.-W.Z. and H.-M.J. analyzed the data; T.Z. and Z.-M.Z. wrote the paper.

Acknowledgments: This work was financially supported by the CAMS Innovation Fund for Medical Sciences (CIFMS, 2016-I2M-1-010 and 2016-I2M-2-003), the Chinese National S\&T Special Project on Major New Drug Innovation (2017ZX09301059), and the National Key Research and Development Program of China (2017YFD0201400-2).

Conflicts of Interest: There is no conflict of interest associated with the authors of this paper.

\section{References}

1. Mabberley, D.J. Mabberley's Plant Book, 3rd ed.; Cambridge University Press: Cambridge, UK, $2008 ;$ p. 154.

2. Zhang, J.P.; Wang, G.W.; Tian, X.H.; Yang, Y.X.; Liu, Q.X.; Chen, L.P.; Li, H.L.; Zhang, W.D. The genus Carpesium: A review of its ethnopharmacology, phytochemistry and pharmacology. J. Ethnopharmacol. 2015, 163, 173-191. [CrossRef] [PubMed]

3. Yang, C.; Yuan, C.S.; Jia, Z.J. Xanthanolides, germacranolides, and other constituents from Carpesium longifolium. J. Nat. Prod. 2003, 66, 1554-1557. [CrossRef] [PubMed]

4. Xie, W.D.; Wang, X.R.; Ma, L.S.; Li, X.; Row, K.H. Sesquiterpenoids from Carpesium divaricatum and their cytotoxic activity. Fitoterapia 2012, 83, 1351-1355. [CrossRef] [PubMed]

5. Gao, X.; Lin, C.J.; Jia, Z.J. Cytotoxic germacranolides and acyclic diterpenoides from the seeds of Carpesium triste. J. Nat. Prod. 2007, 70, 830-834. [CrossRef] [PubMed]

6. Lin, Y.L.; Ou, J.C. Napalolides A-D, four new sesquiterpene lactones from Carpesium nepalense. J. Nat. Prod. 1996, 59, 991-993. [CrossRef]

7. Li, X.W.; Weng, L.; Gao, X.; Zhao, Y.; Pang, F.; Liu, J.H.; Zhang, H.F.; Hu, J.F. Antiproliferative and apoptotic sesquiterpene lactones from Carpesium faberi. Bioorg. Med. Chem. Lett. 2011, 21, 366-372. [CrossRef] [PubMed]

8. Yang, Y.X.; Shan, L.; Liu, Q.X.; Shen, Y.H.; Zhang, J.P.; Ye, J.; Xu, X.K.; Li, H.J.; Zhang, W.D. Carpedilactones A-D, four new isomeric sesquiterpene lactone dimers with potent cytotoxicity from Carpesium faberi. Org. Lett. 2014, 16, 4216-4219. [CrossRef] [PubMed]

9. Wu, J.W.; Tang, C.P.; Chen, L.; Qiao, Y.; Geng, M.Y.; Ye, Y. Dicarabrones A and B, a pair of new epimers dimerized from sesquiterpene lactones via a [3 + 2] cycloaddition from Carpesium abrotanoides. Org. Lett. 2015, 17, 1656-1659. [CrossRef] [PubMed] 
10. Editorial Committee of the Administration Bureau of Traditional Chinese Medicine. Chinese Materia Medica; Shanghai Science \&Technology Press: Shanghai, China, 1999; Volume 21, p. 761.

11. Kim, E.J.; Jin, H.K.; Kim, Y.K.; Lee, H.Y.; Lee, S.Y.; Lee, K.R.; Zee, O.P.; Han, J.W.; Lee, H.W. Suppression by a sesquiterpene lactone from Carpesium divaricatum of inducible nitric oxide synthase by inhibiting nuclear factor- $\mathrm{KB}$ activation. Biochem. Pharmacol. 2001, 61, 903-910. [CrossRef]

12. Zee, O.P.; Kim, D.K.; Choi, S.U.; Lee, C.O.; Lee, K.R. A new cytotoxic acyclic diterpene from Carpesium divaricatum. Arch. Pharm. Res. 1999, 22, 225-227. [CrossRef] [PubMed]

13. Zee, O.P.; Kim, D.K.; Lee, K.R. Thymol derivatives from Carpesium divaricatum. Arch. Pharm. Res. 1998, 21, 618-620. [CrossRef] [PubMed]

14. Chung, I.M.; Seo, S.H.; Kang, E.Y.; Park, W.H.; Park, S.D.; Moon, H.I. Antiplasmodial activity of isolated compounds from Carpesium divaricatum. Phytother. Res. 2010, 24, 451-453. [CrossRef] [PubMed]

15. Kim, D.K.; Beak, N.I.; Choi, S.U.; Lee, C.O.; Lee, K.R.; Zee, O.P. Four new cytotoxic germacranolides from Carpesium divaricatum. J. Nat. Prod. 1997, 60, 1199-1202. [CrossRef] [PubMed]

16. Maruyama, M. Sesquiterpene lactones from Carpesium divaricatum. Phytochemistry 1990, 29, 547-550. [CrossRef]

17. Kim, D.K.; Lee, K.R.; Zee, O.P. Sesquiterpene lactones from Carpesium divaricatum. Phytochemistry 1997, 46, 1245-1247. [CrossRef]

18. Lee, H.J.; Jung, H.; Kwon, J.; Li, H.; Lee, D.Y.; Lim, H.J.; Kim, M.R.; Moon, D.C.; Ryu, J.H. A germacranolide sesquiterpene lactone suppressed inducible nitric oxide synthase by downregulating NF- $\mathrm{B}$ activity. Can. J. Physiol. Pharmacol. 2011, 89, 232-237. [CrossRef] [PubMed]

19. Moon, H.I.; Zee, O. Antiproliferative effect from sesquiterpene lactones of Carpesium rosulatum MlQ consumed in South Korea on the five human cancer cell lines. Rec. Nat. Prod. 2010, 4, 3149-3155.

20. Moon, H.I.; Zee, O. Sesquiterpene lactones from Carpesium rosulatum with potential cytotoxicity against five human cancer cell lines. Hum. Exp. Toxicol. 2010, 30, 1083-1087. [CrossRef] [PubMed]

21. Baruah, R.N.; Sharma, R.P. Thyagarajan, G. Unusual germacranolides from Inula eupatorioides. J. Org. Chem. 1980, 45, 4838-4843. [CrossRef]

22. Baruah, N.C.; Baruah, R.N.; Sharma, R.P.; Baruah, J.N. Germacranolides of Inula eupatorioides. 2. Absolute configuration of the ineupatorolides. J. Org. Chem. 1982, 47, 137-140. [CrossRef]

23. Goswami, A.C.; Baruah, R.N.; Sharma, R.P.; Baruah, J.N.; Kulanthaivel, P.; Herz, W. Germacranolides from Inula cappa. Phytochemistry 1984, 23, 367-372. [CrossRef]

24. Wang, F.Y.; Li, X.Q.; Sun, Q.; Yao, S.; Ke, C.Q.; Tang, C.P.; Liu, H.C.; Geng, M.Y.; Ye, Y. Sesquiterpene lactones from Carpesium divaricatum. Phytochem. Lett. 2012, 5, 639-642. [CrossRef]

25. Gonzalez, A.G.; Barrera, J.B.; Mendez, J.T.; Martinez, J.E.; Sanchez, M.L. Germacranolides from Allagopappus viscosissimus. Phytochemistry 1992, 31, 330-331. [CrossRef]

26. Gonzalez, A.G.; Bermejo, J. Sesquiterpene lactones and other constituents of Allagopappus species. J. Nat. Prod. 1995, 58, 432-437. [CrossRef]

27. Zhang, T.; Si, J.G.; Zhang, Q.B.; Ding, G.; Zou, Z.M. New highly oxygenated germacranolides from Carpesium divaricatum and their cytotoxic activity. Sci. Rep. 2016, 6, 27237. [CrossRef] [PubMed]

28. Wang, B.J.; Won, S.J.; Su, Z.R.; Yu, C.L. Free radical scavenging and apoptotic effects of cordyceps sinensis fractionated by supercritical carbon dioxide. Food Chem. Toxicol. 2005, 43, 543-552. [CrossRef] [PubMed]

Sample Availability: Samples of the compounds $\mathbf{1}$ and $\mathbf{5}$ are available from the authors. 